\title{
Electrically isolated SiGe quantum dots
}

\author{
Emma Tevaarwerk, P. Rugheimer, O. M. Castellini, D. G. Keppel, S. T. Utley, \\ D. E. Savage, M. G. Lagally, and M. A. Eriksson ${ }^{\text {a) }}$ \\ University of Wisconsin-Madison, Madison, Wisconsin 53706
}

(Received 30 January 2002; accepted for publication 11 April 2002)

\begin{abstract}
A variation of electric force microscopy (EFM) is used to measure the electrical isolation of SiGe quantum dots (QDs). The SiGe QDs are grown on mesas of ultrathin silicon on insulator. Near the mesa edges, the thin silicon layer has been incorporated into the QDs, resulting in electrically isolated QDs. Away from the edges, the silicon layer is not incorporated and has a two-dimensional resistivity of less than $800 \mathrm{~T} \Omega$ per sq, resulting in relatively short $R C$ times for charge flow on the mesa. The EFM technique we use here is a powerful probe of samples and devices with floating-gate geometries. (C) 2002 American Institute of Physics. [DOI: 10.1063/1.1484251]
\end{abstract}

Electrically isolated quantum dots (QDs) are promising candidates for charge storage in future silicon nanoelectronics. For example, QDs could be used as patterned gates for nonvolatile floating gate memories. ${ }^{1}$ Long storage times have recently been demonstrated for nanocrystals embedded in silicon dioxide. ${ }^{2,3}$ Random variations in the vertical position of QDs within the oxide may result in fluctuations in the charging voltages of such devices. ${ }^{4,5}$ A method for fabricating electrically isolated QDs with well-controlled vertical positions is therefore desirable.

When QDs self-assemble in the Stranski-Krastanov growth process, the underlying substrate plays an active role in determining the quantum dot properties. ${ }^{6,7}$ In addition to the well-known effects of strain, substrate atoms can be incorporated into self-assembled SiGe quantum dots during growth. ${ }^{6,8}$ Such dot-substrate interactions suggest that QD properties could be modified using engineered substrates. For example, during growth on ultrathin silicon-on-insulator (SOI) a limited amount of silicon is available for incorporation into the QDs. Such substrates could provide a way in which to fabricate electrically isolated QDs with wellcontrolled vertical positions.

In this letter, we report the self-assembly of electrically isolated $\mathrm{SiGe}$ QDs on ultrathin SOI patterned into mesas. We show that electric force microscopy (EFM) is a sensitive probe of very large resistivities in floating-gate samples. The resistivity in regions between these QDs decreases as a function of distance from the mesa edge. This decreased resistivity results from reduced incorporation of the underlying $\mathrm{Si}$ layer into the QDs. Near the center of the mesa, the ultrathin silicon layer remains unincorporated and behaves as a conducting layer in EFM measurements. Near the edge of the mesa, the ultrathin silicon layer is entirely consumed by the surrounding QDs, resulting in electrically isolated QDs. The EFM technique we use here may be extended to study inplane resistivity in a wide variety of floating-gate samples.

A SOI wafer with $10^{15}$ boron acceptors $/ \mathrm{cm}^{3}$ is thinned by thermal oxidation until roughly $10-15 \mathrm{~nm}$ of silicon remains. This ultrathin SOI wafer is patterned and etched, and isolated silicon mesas of $10 \mu \mathrm{m}$ lateral dimensions are cre-

${ }^{a)}$ Electronic mail: maeriksson@ @acstaff.wisc.edu ated. Germanium $(1.5 \mathrm{~nm})$ is deposited onto the patterned SOI at $700{ }^{\circ} \mathrm{C}$ by molecular beam epitaxy. Figure 1 (a) shows an intermittent-contact atomic force microscopy (IC-AFM) image of the sample surface immediately after deposition, and Fig. 1(b) is a schematic cross-section of the sample geometry. Because of thermodynamic and kinetic restrictions, the SiGe QDs first nucleate at the edge of the mesa and then continue to grow in concentric rings toward the center of the mesa. ${ }^{6}$ By halting Ge deposition at an early stage, only four or five rings nucleate near the edge of the mesa [Fig. 1(a)]. These are the "edge QDs" whose electrical isolation is discussed in this letter. The "islands" that appear in the center of the mesa are very different, and are discussed elsewhere. ${ }^{10}$ Transmission electron micrographs (not shown) reveal the absence of a Si template next to edge QDs. ${ }^{10}$

As we show in this letter, EFM provides an excellent measure of the degree of electrical isolation (i.e., in-plane resistivity) of samples with floating-gate geometries. EFM is sensitive to gradients in the electrostatic forces on a conduct-

(a)

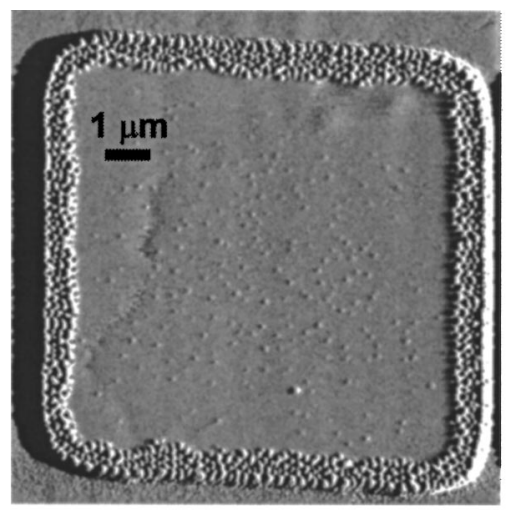

(b)

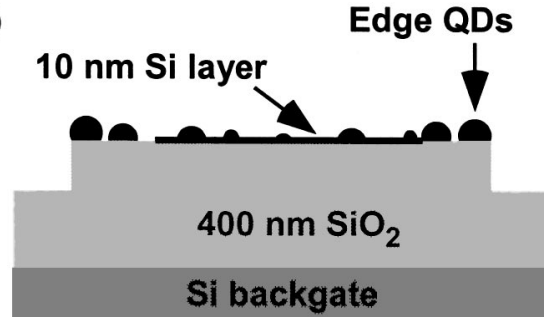

FIG. 1. (a) IC-AFM image and (b) schematic diagram of a $10 \mu \mathrm{m}$ mesa after growth. 
(a)

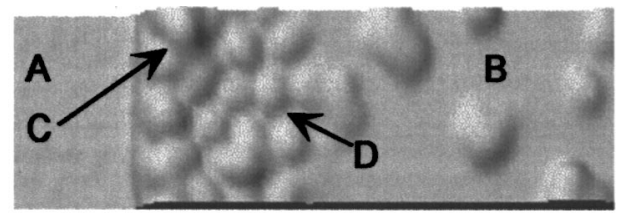

$500 \mathrm{~nm}$

(b)

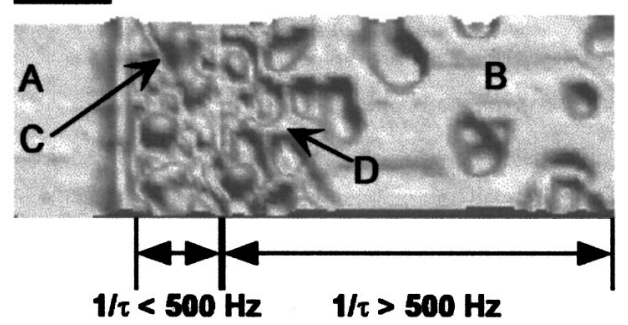

FIG. 2. (a) Topography and (b) corresponding $\Delta \nu_{2 \omega}$ of the left quarter of a $10 \mu \mathrm{m}$ mesa. Regions of fast and slow $R C$ times $(\tau)$ are delineated. Data are acquired with a modified Digital Instruments (DI) Multimode AFM and Nanoscope IIIa controller. Images are acquired in two scans, first by an IC-AFM scan $\left(V_{\text {tip }}=0\right)$ to determine the topography and then an EFM scan. During the EFM scan, the conducting tip floats $50 \mathrm{~nm}$ above the sample with a voltage $V_{\text {tip }}=2 \mathrm{~V}+2 \mathrm{~V} \sin (2 \pi \times 500 \mathrm{~Hz} \times t)$.

ing tip as it floats over the sample. For very small amplitudes of vibration the shift in resonance frequency $\Delta v$ is approximately

$$
\Delta v \approx-\frac{v_{0}}{2 k}\left(\frac{\partial F}{\partial z}\right)
$$

where $k$ is the spring constant, $v_{0}$ the resonance frequency of the cantilever, and $F$ the force on the tip. ${ }^{11,12}$ Factors such as the local work function, trapped charge, dielectric constant, and geometry all influence the frequency shift measured.

In our samples, the region of interest is a floating layer between the tip and back-gate, specifically the QDs and remaining Si layer depicted in Fig. 1(b). In order to focus on the in-plane conductivity of this layer, we apply an ac voltage to the tip with respect to the back gate, $V_{\text {tip }}=V_{\mathrm{dc}}$ $+V_{\text {ac }} \sin (\omega t)$. This produces a frequency shift $\Delta \nu$ with Fourier components at dc, $\omega$, and $2 \omega$. We measure the resonance frequency shift component at $2 \omega$,

$$
\Delta \nu_{2 \omega} \propto-\frac{\partial^{2} C}{\partial z^{2}} \times V_{\mathrm{ac}}^{2}
$$

where $C$ is the tip-sample capacitance, and $z$ is the tipsample separation. ${ }^{13}$ Thus, $\Delta \nu_{2 \omega}$ is a measure of anything that influences the tip-sample capacitance, such as the local in-plane conductivity of the floating layer. The EFM signal $\Delta \nu_{2 \omega}$ is not sensitive to differences in work function or local trapped charges, ${ }^{13,14}$ and is therefore an excellent probe of samples with floating-gate geometries.

Figure 2 shows an IC-AFM image and corresponding frequency shift $\Delta \nu_{2 \omega}$ of the left quarter of a $10 \mu \mathrm{m}$ mesa. In the interior of the mesa (region B) $\Delta \nu_{2 \omega}$ is $30 \mathrm{~Hz}$; off the mesa (region A) $\Delta \nu_{2 \omega}$ is $6 \mathrm{~Hz}$. This observation is at first surprising. One would naively expect regions off the mesa (close to the back gate) to have a stronger electric field gradient than regions on the mesa $(125 \mathrm{~nm}$ farther from the back gate).

The larger electric field gradient in region B can be understood, as we show here, if the thin silicon layer on the Downloaded 19 Feb 2007 to 128.104.198.19. Redistribution subject

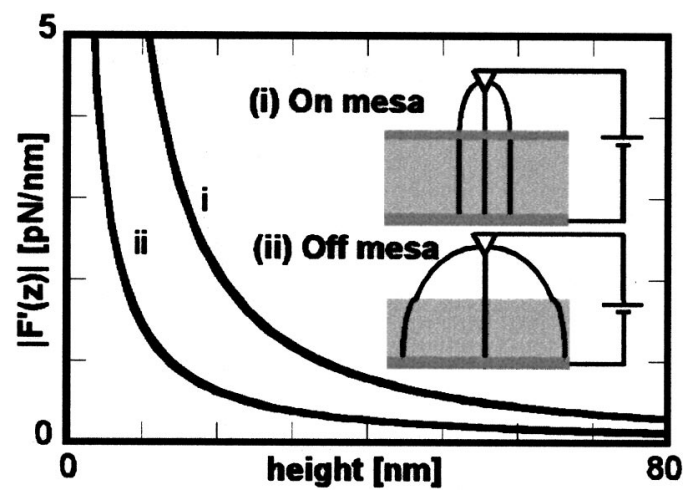

FIG. 3. Calculated $F^{\prime}(z)$ vs height $z$. Curve (i) corresponds to the tip over the mesa and curve (ii) corresponds to the tip off the mesa. Inset: Schematic of the geometry of (i) and (ii). Note the very different behavior of the field lines on and off the mesa. Calculation parameters: On mesa, the oxide thickness is $400 \mathrm{~nm}$ and is capped by a $10 \mathrm{~nm}$ thick conducting layer. Off mesa, the oxide thickness is $275 \mathrm{~nm}$ and is not capped. The tip has a radius of curvature of $25 \mathrm{~nm}$.

mesa acts as a conducting plane. Figure 3 (inset) shows tipsample geometries both when the tip is (i) on the mesa over a thin conducting layer and (ii) off the mesa over only oxide. On the mesa, all of the bending of the electric field lines occurs between the tip and thin conducting layer. Off the mesa, where there is no conducting layer, the field lines bend over the entire distance between the tip and back gate. Thus, the presence of a thin conducting layer results in a larger electric field gradient (EFM frequency shift) on the mesa than off.

Figure 3 shows the calculated tip-sample force gradient for both the (i) on and (ii) off mesa geometries. ${ }^{15}$ The calculations use the full three-dimensional (3D) $\mathrm{MESP}^{16}$ cantilever and tip geometry; the sample geometry is simplified as shown in the inset. The calculated force gradient on the mesa is indeed larger than the calculated force gradient off the mesa. At a tip-sample separation of $50 \mathrm{~nm}$ the ratio of the frequency shifts on and off the mesa is $3: 1$. This value is remarkably close to the 5:1 ratio in Fig. 2, given the simplified sample geometry used in the calculation.

In our experiments, the thin layer of silicon acts as a conductor. That is, the $R C$ time constant $(\tau)$ of the thin $\mathrm{Si}$ layer is faster than the applied ac frequency, $500 \mathrm{~Hz}$, so that charge oscillates beneath the tip in phase with the ac voltage applied. The exact origin of the carriers and their conduction mechanism is uncertain, although surface traps are likely involved. ${ }^{17}$ Assuming a tip-sample capacitance of $3 \mathrm{aF},{ }^{18}$ the effective two-dimensional (2D) resistivity of the Si layer is $\rho_{2 \mathrm{D}} \leqslant 800 \mathrm{~T} \Omega$ per sq. It is useful to note that this $2 \mathrm{D}$ resistivity corresponds to a 3D resistivity, $\rho_{3 \mathrm{D}} \leqslant 8 \times 10^{6} \Omega \mathrm{m}$. For comparison, the original doped silicon wafer had resistivity $\rho=0.2 \Omega \mathrm{m}$, more than $10^{7}$ times smaller than the $\rho_{3 \mathrm{D}}$ measured. Thus, although the floating silicon layer is almost certainly depleted by its surfaces, its residual conductivity is sufficient to be measured by EFM. For samples with floating-gate geometries, even the presence of weakly conducting materials beneath the tip has a significant effect on the EFM frequency shifts measured.

Figure 2 shows that the frequency shift $\Delta \nu_{2 \omega}$ in region C between edge QDs is nearly identical to that off the mesa. This is because there is no silicon between these QDs. In to AIP license or copyright, see http://apl.aip.org/apl/copyright.jsp 
fact, all the QDs at the edge are surrounded by regions of low frequency shift. Using the same $R C$ time constant argument as that above, the edge quantum dots are isolated from each other by $\rho_{2 \mathrm{D}}>800 \mathrm{~T} \Omega$ per sq.

In contrast, slightly farther from the edge between QDs in region $\mathrm{D}, \Delta \nu_{2 \omega}$ is almost identical to that in region $\mathrm{B}$. The AFM image in Fig. 2(a) shows that region D has topography very similar to region $\mathrm{C}$. Thus, the transition from small $\Delta \nu_{2 \omega}$ in region $\mathrm{C}$ to large $\Delta \nu_{2 \omega}$ in region $\mathrm{D}$ is predomintly due to the presence of the silicon layer in region D. In fact, from the calculations in Fig. 3, curve (i), we determine that more than half $(56 \%)$ of the tip-sample force gradient arises from interactions between the sample and the $25 \mathrm{~nm}$ of the tip closest to the sample. Thus, it is not surprising that EFM has sufficient resolution to observe the electrical isolation of the QDs in Fig. 2.

We use an ac voltage at $\omega=2 \pi \times 500 \mathrm{~Hz}$ to determine a transition between regions with $R C$ times faster and slower than this frequency. By varying the frequency $\omega$ of the ac voltage applied to the tip, it may be possible to identify regions with faster or slower response times. This technique can be used to measure in-plane resistivities in samples with floating-gate geometries, provided electrical contact can be made to a back-gate.

EFM measurements demonstrate that electrically isolated SiGe QDs can be grown using ultrathin SOI. This fabrication technique offers good control of the vertical position of the QDs, which may be useful for nanocrystal flash memory. Although seldom exploited, the $\Delta \nu_{2 \omega}$ response in EFM is a powerful probe of samples with floating-gate geometries. $2 \omega$-EFM measures very large resistivities, and is thus a good way to test for nanoscale electrical isolation.
This research was supported by the NSF CAREER (DMR-0094063) and MRSEC (DMR-9632527) programs, the Research Corporation (RI0588), and by the NSA and ARDA under ARO Contract No. DAAD19-01-1-0515.

${ }^{1}$ S. Tiwari, F. Rana, H. Hanafi, A. Hartstein, E. F. Crabbé, and K. Chan, Appl. Phys. Lett. 68, 1377 (1996).

${ }^{2}$ E. A. Boer, M. L. Brongersma, H. A. Atwater, R. C. Flagan, and L. D. Bell, Appl. Phys. Lett. 79, 791 (2001).

${ }^{3}$ D. M. Schaadt, E. T. Yu, S. Sankar, and A. E. Berkowitz, Appl. Phys. Lett. 74, 472 (1999).

${ }^{4}$ G. Iannaccone and P. Coli, Appl. Phys. Lett. 78, 2046 (2001).

${ }^{5}$ Y.-C. King, T.-J. King, and C. Hu, IEEE Trans. Electron Devices 48, 696 (2001).

${ }^{6}$ A. R. Woll, P. Rugheimer, and M. G. Lagally, Int. J. High Speed Electron. Syst. (in press).

${ }^{7}$ P. Schittenhelm, C. Engel, F. Findeis, G. Abstreiter, A. A. Darhuber, G. Bauer, A. O. Kosogov, and P. Werner, J. Vac. Sci. Technol. B 16, 1575 (1998).

${ }^{8}$ S. A. Chapparo, Y. Zhang, and J. Drucker, Appl. Phys. Lett. 76, 3534 (2000).

${ }^{9}$ W. L. Henstrom, C.-P. Liu, J. M. Gibson, T. I. Kamins, and R. S. Williams, Appl. Phys. Lett. 77, 1623 (2000).

${ }^{10}$ F. Lui, P. Rugheimer, E. Mateeva, D. E. Savage, and M. G. Lagally, Nature (London) 416, 498 (2002)

${ }^{11}$ Y. Martin, C. C. Williams, and H. K. Wickramasinghe, J. Appl. Phys. 61, 4723 (1987)

${ }^{12}$ T. R. Albrecht, P. Grütter, D. Home, and D. Rugar, J. Appl. Phys. 69, 668 (1991).

${ }^{13}$ B. D. Terris, J. E. Stern, D. Rugar, and H. J. Mamin, Phys. Rev. Lett. 63, 2669 (1989)

${ }^{14}$ T. D. Krauss and L. E. Brus, Phys. Rev. Lett. 83, 4840 (1999).

${ }^{15}$ The Laplace equation for the full three-dimensional tip-sample geometry was solved using FLEXPDE version 2.22, PDE Solutions Inc., P.O. Box 4217, Antioch, CA 94531

${ }^{16}$ MESP tip, Digital Instruments, 112 Robin Hill Road, Santa Barbara, CA 93117.

${ }^{17}$ H. Fujii, S. Kanemaru, T. Matsukawa, and J. Itoh, Appl. Phys. Lett. 75, 3986 (1999).

${ }^{18}$ To calculate the order of magnitude of the tip-sample capacitance, the tip is approximated as a sphere with radius of $25 \mathrm{~nm}$ at a distance $50 \mathrm{~nm}$ from a plane. 